\title{
The role of the programmed cell death protein-1/programmed death-ligand 1 pathway, regulatory $T$ cells and $T$ helper 17 cells in tumor immunity: a narrative review
}

\author{
Lanfang Zhang^, Mingjuan Zhang, Jinxiu Xu, Shan Li, Yu Chen, Wenjing Wang, Juntian Yang, \\ Shengyun Li, Meiling Gu \\ Department of Chemotherapy Unit 2, Weihai Municipal Hospital, Cheeloo College of Medicine, Shandong University, Weihai, China \\ Contributions: (I) Conception and design: L Zhang; (II) Administrative support: None; (III) Provision of study materials or patients: All authors; \\ (IV) Collection and assembly of data: L Zhang; (V) Data analysis and interpretation: L Zhang; (VI) Manuscript: All authors; (VII) Final approval of \\ manuscript: All authors. \\ Correspondence to: Lanfang Zhang, PhD. Department of Chemotherapy Unit 2, Weihai Municipal Hospital, 248 Tongyi Road, Weihai 264200 , China. \\ Email: zlfxueye@163.com.
}

\begin{abstract}
Tumor immunotherapy, especially that involving programmed cell death protein-1 (PD-1)/ programmed death-ligand 1 (PD-L1) immunosuppressive checkpoint inhibitors, has become an important part of tumor treatment strategy in the past decade. Blocking PD-1/PD-L1 signaling pathway can reduce the inhibitory effect of PD-1 pathway on T cells, promote the anti-tumor activity of activated $\mathrm{T}$ cells, and prolong the remission period of tumor. While PD-1/PD-L1 immunotherapy is effective in the treatment of solid malignant tumors, it also has shortcomings, due to the complexity of the tumor microenvironment (TME). Regulatory T cells (Tregs) and T helper 17 (Th17) cells play an important role in the TME and are closely related to the occurrence and development of tumors. Tregs can inhibit the anti-tumor immune effect, while Th17 cells play a dual role in tumor immunity, which not only promotes tumorigenesis but also promotes anti-tumor immunity. In the occurrence and development of tumor, PD-1/PD-L1 pathway, Tregs and Th17 cells are interrelated. However, the complicated relationship between the PD-1/PD-L1 pathway, Tregs, and Th17 cells has not been fully clarified. Here, we summarize the immunoregulation mechanisms and discuss the crosstalk between the PD-1/PD-L1 pathway, Tregs, and Th17 cells, with the aim of providing novel insights for future cancer treatment.
\end{abstract}

Keywords: Tumor; programmed cell death protein-1 (PD-1)/programmed death-ligand 1 (PD-L1); T helper 17 cells (Th17 cells); regulatory T cells (Tregs)

Submitted Sep 10, 2020. Accepted for publication Nov 06, 2020.

doi: 10.21037/atm-20-6719

View this article at: http://dx.doi.org/10.21037/atm-20-6719

\section{Introduction}

The emergence of immune checkpoint inhibitor (ICI)targeted immunotherapy has greatly and successfully changed the treatment landscape for several advanced malignant tumors. The mechanism of immunocheckpoint blockers is different from chemotherapy and targeted therapy. The mechanism of ICI is targeted at tumor immune response, which can prolong the remission period after treatment. Programmed cell death protein-1 (PD-1)/ programmed death-ligand 1 (PD-L1) blocker is the most widely used ICI in clinical practice. It breaks the immune tolerance of the body to tumor by blocking the PD-1/ PD-L1 pathway and effectively enhances the anti-tumor

^ ORCID: 0000-0003-3159-9004. 
immunity.

The interaction between PD-1 and PD-L1 plays a role in the regulation of both regulatory $\mathrm{T}$ cells (Tregs) and $\mathrm{T}$ helper 17 (Th17) cells. As an important type of suppressive immune cell in the TME, Tregs have crucial involvement in the occurrence and development of tumors. The number and function of Tregs and Th17 cells influence the blocking effect of PD-1/PD-L1 inhibitors and are vital to tumor development. Meanwhile, Th17 cells in TME play a dual role in tumor development. The roles of the PD-1/PDL1 pathway, Tregs, and Th17 cells in tumor occurrence and development are intertwined; however, this complex relationship has yet to be fully explored. Herein, we summarize the immunoregulation mechanisms of the PD-1 pathway, Tregs, and Th17 cells, and examine the crosstalk between the three in tumor progression. We present the following article in accordance with the Narrative Review reporting checklist (available at http://dx.doi.org/10.21037/ atm-20-6719).

\section{The PD-1/PD-L1 pathway and tumor immune escape}

PD-1, which belongs to the immunoglobulin superfamily, is a 288 amino-acid membrane protein and an important immunosuppressive molecule. PD-1 was originally cloned from the apoptotic mouse T cell hybridoma 2B4.11 (1). It's binding leads to cell cycle blockade but not cell death. PD-1 is expressed on multiple immunocytes, including activated T cells, B cells, natural killer cells (NKs), monocytes, and dendritic cells (DCs) $(2,3)$. The expression of PD-1 on the surface of activated $\mathrm{T}$ cells occurs during the initial activation phase.

$\mathrm{PD}-1$ recognizes two ligands, $\mathrm{PD}-\mathrm{L} 1$ and programmed death-ligand 2(PD-L2, B7-DC or CD273). PD-L1 is expressed by a wide range of cells, including $\mathrm{T}$ cells, $\mathrm{B}$ cells, Tregs, antigen-presenting cells (APCs), vascular endothelial cells, mesenchymal stem cells, reticular fibroblasts, and islet cells. PD-L1 has also been detected in immune privilege sites such as the eyes, placenta, and testes (4). Moreover, PD-L1 is highly expressed on tumor cells. Compared with PD-L1, the expression range of PD-L2 was narrower, mainly in macrophages and dendritic cells. The PD-L1/PD-1 signaling pathway has been identified as a contributor to tumor immune escape $(5,6)$. The $\mathrm{C}$ and $\mathrm{N}$-terminal amino acid residues of the intracellular domain of PD-1 have two independent phosphorylation sites: the immunoreceptor tyrosine-based inhibitory motif
(ITIM) and the immunoreceptor tyrosine-based switch motif (ITSM) (7). ITSM plays an important role in the immunosuppression of PD-1. The combination of PD-1 and PD-L1 can phosphorylate ITSM and activate intracellular pathways, which ultimately plays an immunosuppressive role. The inhibition mechanism of PD-1 / PD-L1 pathway on $\mathrm{T}$ and $\mathrm{B}$ lymphocytes is different (6). When PD-1 binds to PD-L1 in T cells, SHP-1/2 molecules are recruited into the C-terminal ITSM to dephosphorylate TCR activation signaling and inhibit the downstream PI3K/AKT pathway, thereby reducing the expression of apoptosis-related genes and inhibiting the cytokine secretion by T lymphocytes (8). Further, the PD-1/PD-L1 pathway can influence the RasERK pathway to inhibit T-cell proliferation (9). In B cells, PD-1 also shows an inhibitory effect. PD-1 can inhibit the $B$ cell activation and weaken the immune response of B lymphocytes to antigens. Following PD-1 activation, SHP-2 molecules are recruited to the C-terminal of PD-1 to dephosphorylate BCR signaling molecules, such as Ig $\alpha /$ $\beta$ or $\mathrm{S} \gamma \mathrm{K}$, therefore desphosphorylating ERK, PI3K, and PLC $\gamma 2$, leading to acute $\mathrm{Ca} 2+$ disorder and the stagnation of B-cell growth $(10,11)$.

The binding of PD-1 to its ligands triggers inhibitory signals involved in the regulation of central and peripheral tolerance through the inhibition of T-cell proliferation, cytokine synthesis, and cytotoxic activity (12). PD-1/ PD-L1 pathway regulates and maintains the tolerance of peripheral CD4 + T cells through a variety of ways, such as maintaining the stability and integrity of lymphocytes. Increasing evidence from animal models and clinical data shows that PD-1 plays an important role in limiting antitumor $T$ cell responses. After the expression of the PD-L1 gene, tumorigenesis and invasiveness are also be enhanced in vivo. In many cancers, the abnormal expression of PD-L1 in tumor cells is related to immune escape, which indicates that the structural differences of PD-L1 are crucial to the development of tumors (13). PD-L1 may participate in cancer cell apoptosis through two mechanisms: congenital resistance and adaptive resistance. Overexpression of PDL1, promoted by oncogenic and constitutive activation signals, can trigger innate (tumor cell innate) resistance (14-18). PD-L1 is not only expressed in tumors, but is also expressed in immune cells (macrophages, DC, myeloid suppressor cells, and lymphocytes) of the TME through inflammatory signaling, which is considered as adaptive resistance $(19,20)$. Previous studies have shown that the expression of PD-L1 is related to an enhanced anti-tumor immune response, which is associated with improved 
prognosis in tumor patients.

PD-1/PD-L ICI therapy can restore the number of effector $\mathrm{T}$ cells (Teffs), promote their cytotoxic immune response against chemotherapy-resistant tumors, restore the activity of CD8 $+\mathrm{T}$ cells depleted in chronic viral infection (21), promote the synthesis of pro-inflammatory cytokines, and restore sustained immunity to tumors (22-25). ICI therapy can enhance the body's natural defense against tumors by promoting a T-cell-specific immune response. ICI therapy has lower toxicity than standard chemotherapy, but it can also result in immunerelated adverse events (irAEs). After blocking the immune checkpoint, the immune tolerance will change and induce iraes. In long-term immune activation, the misdirected stimulation of the immune system toward a normal tissue can lead to autoimmune-like or inflammatory side effects. Even after anti-PD-1 antibody treatment is stopped, delayed autoimmune toxicity can even occur as time passes. Therefore, in view of the rapid increase in the number of patients receiving treatment with anti-PD-1 agents, longterm follow-up is necessary in order to observe the possible delayed adverse reactions after treatment. There is no unified standard for the upper limit of the duratio16n of ICI use except for the occurrence of disease progression, intolerance or death of patients, and the optimal treatment time of ICI is still an outstanding issue.

\section{Tregs and suppression of antitumor immunity}

In 1970, Gershon and Kondo discovered a suppressor $T$ cell population that could down-regulate the immune response (26). Owing to the observations of Sakaguchi's group (27), CD4+CD25+ Treg cells have been one of the most important research focuses in the field of immunology in the last 20 years. The transcription factor fork-head box P3 (Foxp3) is specifically expressed in CD4+CD25+Treg cells. Based on the developmental process, Tregs have been categorized into two major groups: natural Tregs (nTregs) and inducible Tregs (iTregs) (28). nTregs are generated in the thymus and are mitotically quiescent in basal conditions (29). The expansion of nTregs requires antigen stimulation, while TCR engagement is unnecessary in the inhibitory activity of nTregs (30). In contrast, iTregs develop from conventional CD4+ T (Tconv) cells in the periphery and can be generated in vitro. Antigenic TCR stimulation, costimulatory molecules, and cytokines, such as transforming growth factor-beta 1 (TGF- $\beta 1)$ and
Interleukin-2 (IL-2), promote the development of iTreg cells (31-33). Tregs inhibit T-cell immunity and avoid the damage caused by $\mathrm{T}$ cell overactivation. Therefore, Tregs plays an important role in maintaining immune homeostasis and self-tolerance (34).

FoxP3+Tregs were reported to significantly inhibit the activation, proliferation and/or effector functions of other CD4+,CD8+ T cells, NK-like cells, NK T cells, B cells and DCs (35). The immunosuppressive mechanism of Tregs is not fully understood. CD4+CD25+Foxp3+Tregs are generally believed to down-regulate immune responses in multiple ways. This includes cell contactdependent suppression, functional modification $(36,37)$ and the secretion of immunosuppressive cytokines (38). Sakaguchi et al. suggested that each type of Treg may have a common core inhibition mechanism or several other complementary mechanisms (39). Another possibility is that multiple inhibition mechanisms coordinate and cooperate with each other. Therefore, the dysfunction of certain pathways may not be enough to seriously affect immunosuppression. There is increasing evidence to suggest that the immunosuppressive functions of Tregs promote tumor progression by inhibiting anti-tumor immunity (40). The infiltration rate of Tregs in tumor tissues has been found to be higher than that in adjacent tissues (41-43). The enrichment of Tregs in tumor tissues can be achieved by recruitment of Tregs around the tumor and local expansion of Tregs themselves (44). Some soluble molecules in the tumor immunosuppressive microenvironment can also promote Treg expansion $(44,45)$. Tregs are attracted to the tumor bed by vascular endothelial growth factor (VEGF) or chemokines (such as CCL17, CCL22, CCL28, and CXCL12) secreted by tumor cells or immune cells $(44,46)$. Tumor-infiltrating Tregs were reported to be selectively depleted through Fcdependent mechanisms involving Fc $\gamma$-receptor-expressing macrophages within the TME, resulting in an increase in the ratio of CD8+ Teffs/Tregs at tumor sites and Tregs in peripheral blood $(47,48)$. In mouse models of melanoma and colon cancer, a reduction in Tregs can increase the response of anti-tumor $\mathrm{T}$ cells and reduce tumor growth $(49,50)$. Treg depletion can improve anti-tumor immunity and enhance the curative effect of tumor treatment. Moreover, a high proportion of Tregs is associated with poor prognosis, suggesting that Treg-mediated T-cell suppression is an important mechanism for tumor cells to escape the immune response (51-53). 


\section{Role of Th17 cells in cancer progression}

Th17 cells are a subset of $T$ cells that produce IL-17 which is the most important effector produced by Th17 cells and is also the source of Th17 nomenclature. Their differentiation process is controlled by the transcription factors orphan nuclear receptor gamma $\mathrm{t}(\mathrm{ROR} \gamma \mathrm{t})$ and signal transducer and activator of signal transducer and activator of transcription 3 (STAT3) and requires exposure to various cytokines $(54,55)$. A number of studies have demonstrated the important role played by IL-17 in the promotion of tumor growth and invasion (56-61). Tumor-associated cells and fibroblasts secrete a large number of cytokines, and the pro-inflammatory cytokine-rich environment can recruit Th17 cells to the TME (62). Th17 cells in the TME can promote the influx of monocytes/macrophages, DCs, NKs, memory CD4+ and CD8+ T cells through the secretion of the chemokines CCL2 and CCL20 $(63,64)$; however, these cells may not have a direct anti-tumor effect. Moreover, Th17 cells not only recruit CD8+ cytotoxic $\mathrm{T}$ cells, but also promote their activation and expansion $(63,64)$.

It is clear that Th17 cells play a multiple role in tumor immunogenicity, promoting both anti-tumor immunity and tumorigenesis $(63,65-70)$. IL-17A has been found to promote tumor cell growth and proliferation. IL-17A can be produced by both Th17 cells and tumor cells (67). IL-17A promotes angiogenesis by stimulating tumor cells to produce VEGF and promotes tumor invasion and metastasis through matrix metalloproteinase-9 produced by tumor and other cells (67-70). Several recent reports have suggested that Th17 responses may have a therapeutic benefit in improving anti-tumor immunity and survival. In a B16 mouse model of melanoma, adoptive T-cell therapy with tumor-specific Th17 cells prompted strong activation of tumor-specific CD8 $+\mathrm{T}$ cells (which are required for an anti-tumor effect), indicating that Th17-driven inflammation can play a key role in antitumor immunity (71). The induction of Th17 responses in mouse pancreatic cancer models has also been shown to delay tumor growth and improve survival (63). Based on experimental evidence, there is little doubt that Th17 responses can drive tumor progression, invasion, and angiogenesis. On the other hand, it is also obvious from experimental models and clinical studies that Th17 responses can support strong anti-tumor immunity and benefit patient survival. Cantini et al., also using a mouse glioma model, reported a biphasic role of Th17 cells (67). Th17 reactions may be heterogeneous, and different effector functions may produce different results. Currently, the regulation mechanism of Th17 cells in tumors is still limited, and the experimental results are inevitably contradictory; consequently, further study is needed.

\section{Crosstalk between PD-1/PD-L1, Tregs, and Th17 cells in tumors}

PD-1/PD-L1 blockers can target the immune changes caused by the TME and recover tumor immunity. PD-1/ PD-L1 inhibitors have a variety of functions, such as enhancing the cytolytic activity of tumor specific T cells, reducing the production of IL-10, promoting the synthesis of pro-inflammatory cytokines, promoting the existence of TEFs, and reducing the number and inhibitory function of Tregs in tumor site $(24,25,72,73)$. Francisco et al. showed that PD-L1 could convert naive CD4+ T cells to Tregs through the simultaneous upregulation of gene of phosphate and tension homology deleted on chromosome ten (PTEN) and downregulation of AKT, ERK2, and Mtor (74). Tregs express PD-1 and PD-L1, which plays a role in the regulation of $\mathrm{T}$ cell tolerance (75). Although the PD-1/ PD-L1 signaling pathway has been found in Foxp3+ Tregs, the role of PD-1/PD-L1 in regulating Treg function and activity has not been fully elucidated (76). The interaction between PD-L1-expressing DCs and T lymphocytes promotes Treg development (77). Francisco et al. reported that PD-L1 induced the differentiation, maintenance, and function of iTreg cells by sustaining and increasing their expression of Foxp3 (74). In the presence of PDL1-Ig, Foxp3 expression and inhibitory Treg function were found to be increased following the activation of $\mathrm{T}$ lymphocytes (74). Furthermore, PD-1/PD-L1 binding was shown to reduce $\mathrm{T}$ cell production, cytokine release, and survival. These results suggest that the host environment and PD-1 signaling may play an important role in the regulation of Treg development and function. Raimondi et al. observed that the regulated compartmentalization of PD-1 discriminates CD4+CD25+ resting Tregs from activated T cells (78). The PD-1 signaling pathway is also important for maintaining Treg inhibition. Compared with $\mathrm{PD}-1^{\text {hi }} \mathrm{CD} 4+$ Tregs, PD- $1^{\text {low }} \mathrm{CD} 4+$ Tregs showed a higher ability to induce B-cell apoptosis and to inhibit CD4+ helper T cells (Th) (79).

Both Tregs and Th17 cells are thought to have potential plasticity. When Th17 cells are activated under certain conditions, they may lose the ability to secrete IL-17 and obtain the ability to secrete IFN- $\gamma$ in the role 
of pro-inflammatory signal. In doing so, they will gain characteristics similar to those of Th1 cells, also known as ex-th17 cells, which may play a role in enhancing autoimmunity and anti-tumor immunity (80). Interestingly, Tregs can inhibit the proliferation of classical Th1 and Th17 cells, but not that of ex-Th17 cells (81). Under the influence of cytokine, Tregs are able to reacquire Th17like characteristics. Conversely, the transition to Th17 cells acquiring Treg-like characteristics has not been well described. Tumor-infiltrating cells that produce IL-17 and are Foxp3-, transdifferentiate into IL-17 ${ }^{\text {neg }}$ Foxp3 + cells, termed "ex-Th17 Tregs" (81). Tumor-associated conversion between Th17 and Treg cells provides insights for targeting the dynamic change of Th17-Treg cells in cancer immunotherapy.

Recent studies have shown that IL-17 and Th17 cells may play an important role in the efficacy and toxicity of checkpoint-blocking therapy in cancer patients (82). The anti-tumor response is mostly related to autoimmune toxicity, which may be related to the level of IL-17. The role of Th17 cells in checkpoint-blocking therapy has not been fully elucidated. The ultimate goal of immunotherapy is to decouple toxicity from persistent anti-tumor immunity. In future, the Treg/Th17 axis may be effectively regulated to enhance tumor immune function and reduce adverse immune reactions in healthy tissues.

\section{Conclusions}

The emergence of PD-1 blockers as cancer treatment strategies highlights the importance of understanding the role of PD-1 in the regulation of peripheral tolerance. It has been unclear whether most of the success of PD-1 blockers is attributable to the elimination of inhibitory effects of $\mathrm{T}$ cells, thereby enhancing the immunity of $\mathrm{T}$ cells, or to interference with the generation or function of Treg cells, which undermines the potential mechanisms of tumor immune escape. The PD-1 pathway and Tregs have a complex association in the progression and treatment of tumors. The proportion and phenotype of Tregs may serve as biomarkers for predicting the clinical efficacy of PD-1/ PD-L1 antagonists. Additionally, the balance between Th17 cells and Tregs is essential for maintaining immune balance in vivo. Treatment of the Treg/Th17 axis may have a profound impact on the survival of cancer patients. Th17 cells and related cytokines not only promote tumor occurrence, but also inhibit tumor growth. Although the related mechanism has not been determined, the exploration of Th17 cells in tumor immunity at different stages of tumor development is still of research value. Future research in the field of immunotherapy may provide new insights into tumor regression, further improving our ability to use the immune system in the fight against cancer.

\section{Acknowledgments}

Funding: None.

\section{Footnote}

Reporting Checklist: The authors have completed the Narrative Review reporting checklist. Available at http:// dx.doi.org/10.21037/atm-20-6719

Conflicts of Interest: All authors have completed the ICMJE uniform disclosure form (available at http://dx.doi. org/10.21037/atm-20-6719). The authors have no conflicts of interest to declare.

Ethical Statement: The authors are accountable for all aspects of the work in ensuring that questions related to the accuracy or integrity of any part of the work are appropriately investigated and resolved.

Open Access Statement: This is an Open Access article distributed in accordance with the Creative Commons Attribution-NonCommercial-NoDerivs 4.0 International License (CC BY-NC-ND 4.0), which permits the noncommercial replication and distribution of the article with the strict proviso that no changes or edits are made and the original work is properly cited (including links to both the formal publication through the relevant DOI and the license). See: https://creativecommons.org/licenses/by-nc-nd/4.0/.

\section{References}

1. Ishida Y, Agata Y, Shibahara K, et al. Induced expression of PD-1, a novel member of the immunoglobulin gene superfamily, upon programmed cell death. EMBO J 1992;11:3887-95.

2. Linsley PS, Ledbetter JA. The role of the CD28 receptor during $T$ cell responses to antigen. Annu Rev Immunol 1993;11:191-212.

3. Tan KW, Chacko AM, Chew V. PD-1 expression and its significance in tumour microenvironment of hepatocellular carcinoma. Transl Gastroenterol Hepatol 2019;4:51. 
4. Keir ME, Butte MJ, Freeman GJ, et al. PD-1 and its ligands in tolerance and immunity. Annu Rev Immunol 2008;26:677-704.

5. Topalian SL, Taube JM, Anders RA, et al. Mechanismdriven biomarkers to guide immune checkpoint blockade in cancer therapy. Nat Rev Cancer 2016;16:275-87.

6. Jiang X, Wang J, Deng X, et al. Role of the tumor microenvironment in PD-L1/PD-1-mediated tumor immune escape. Mol Cancer 2019;18:10.

7. Zhang X, Schwartz JC, Guo X, et al. Structural and functional analysis of the costimulatory receptor programmed death-1. Immunity 2004;20:337-47.

8. Hofmeyer KA, Jeon H, Zang X. The PD-1/PD-L1 (B7H1) pathway in chronic infection-induced cytotoxic T lymphocyte exhaustion. J Biomed Biotechnol 2011;2011:451694.

9. Patsoukis N, Brown J, Petkova V, et al. Selective effects of PD-1 on Akt and Ras pathways regulate molecular components of the cell cycle and inhibit $\mathrm{T}$ cell proliferation. Sci Signal 2012;5:ra46.

10. Okazaki T, Maeda A, Nishimura H, et al. PD-1 immunoreceptor inhibits $\mathrm{B}$ cell receptor-mediated signaling by recruiting src homology 2-domain-containing tyrosine phosphatase 2 to phosphotyrosine. Proc Natl Acad Sci U S A 2001;98:13866-71.

11. Nicholas KJ, Zern EK, Barnett L, et al. B cell responses to HIV antigen are a potent correlate of viremia in HIV1 infection and improve with PD-1 blockade. PLoS One 2013;8:e84185.

12. Mazanet MM, Hughes CC. B7-H1 is expressed by human endothelial cells and suppresses T cell cytokine synthesis. J Immunol 2002;169:3581-8.

13. Kataoka K, Shiraishi Y, Takeda Y, et al. Aberrant PD-L1 expression through 3 '-UTR disruption in multiple cancers. Nature 2016;534:402-6.

14. Azuma K, Ota K, Kawahara A, et al. Association of PDL1 overexpression with activating EGFR mutations in surgically resected nonsmall-cell lung cancer. Ann Oncol 2014;25:1935-40.

15. Chen N, Fang W, Zhan J, et al. Upregulation of PDL1 by EGFR Activation Mediates the Immune Escape in EGFR-Driven NSCLC: Implication for Optional Immune Targeted Therapy for NSCLC Patients with EGFR Mutation. J Thorac Oncol 2015;10:910-23.

16. Ota K, Azuma K, Kawahara A, et al. Induction of PDL1 Expression by the EML4-ALK Oncoprotein and Downstream Signaling Pathways in Non-Small Cell Lung Cancer. Clin Cancer Res 2015;21:4014-21.
17. Skoulidis F, Byers LA, Diao L, et al. Co-occurring genomic alterations define major subsets of KRAS-mutant lung adenocarcinoma with distinct biology, immune profiles, and therapeutic vulnerabilities. Cancer Discov 2015;5:860-77.

18. Lastwika KJ, Wilson W, 3rd, Li QK, et al. Control of PD-L1 Expression by Oncogenic Activation of the AKTmTOR Pathway in Non-Small Cell Lung Cancer. Cancer Res 2016;76:227-38.

19. Ribas A. Adaptive Immune Resistance: How Cancer Protects from Immune Attack. Cancer Discov 2015;5:9159.

20. Lou Y, Diao L, Cuentas ER, et al. Epithelial-Mesenchymal Transition Is Associated with a Distinct Tumor Microenvironment Including Elevation of Inflammatory Signals and Multiple Immune Checkpoints in Lung Adenocarcinoma. Clin Cancer Res 2016;22:3630-42.

21. Barber DL, Wherry EJ, Masopust D, et al. Restoring function in exhausted CD8 T cells during chronic viral infection. Nature 2006;439:682-7.

22. Hino R, Kabashima K, Kato Y, et al. Tumor cell expression of programmed cell death-1 ligand 1 is a prognostic factor for malignant melanoma. Cancer 2010;116:1757-66.

23. Gatalica Z, Snyder C, Maney T, et al. Programmed cell death 1 (PD-1) and its ligand (PD-L1) in common cancers and their correlation with molecular cancer type. Cancer Epidemiol Biomarkers Prev 2014;23:2965-70.

24. Okiyama N, Katz SI. Programmed cell death 1 (PD-1) regulates the effector function of CD8 T cells via PD-L1 expressed on target keratinocytes. J Autoimmun 2014;53:19.

25. Spranger S, Koblish HK, Horton B, et al. Mechanism of tumor rejection with doublets of CTLA-4, PD-1/PD-L1, or IDO blockade involves restored IL-2 production and proliferation of CD8(+) T cells directly within the tumor microenvironment. J Immunother Cancer 2014;2:3.

26. Gershon RK, Kondo K. Cell interactions in the induction of tolerance: the role of thymic lymphocytes. Immunology 1970;18:723-37.

27. Sakaguchi S, Sakaguchi N, Asano M, et al. Immunologic self-tolerance maintained by activated $\mathrm{T}$ cells expressing IL-2 receptor alpha-chains (CD25). Breakdown of a single mechanism of self-tolerance causes various autoimmune diseases. J Immunol 1995;155:1151-64.

28. Gianchecchi E, Fierabracci A. Inhibitory Receptors and Pathways of Lymphocytes: The Role of PD-1 in Treg Development and Their Involvement in Autoimmunity Onset and Cancer Progression. Front Immunol 
$2018 ; 9: 2374$

29. Kuniyasu $Y$, Takahashi $T$, Itoh $M$, et al. Naturally anergic and suppressive $\mathrm{CD} 25(+) \mathrm{CD} 4(+) \mathrm{T}$ cells as a functionally and phenotypically distinct immunoregulatory $\mathrm{T}$ cell subpopulation. Int Immunol 2000;12:1145-55.

30. Bienvenu B, Martin B, Auffray C, et al. Peripheral CD8+CD25+ T lymphocytes from MHC class II-deficient mice exhibit regulatory activity. J Immunol 2005;175:24653.

31. Boyman O, Sprent J. The role of interleukin-2 during homeostasis and activation of the immune system. Nat Rev Immunol 2012;12:180-90.

32. Malchow S, Leventhal DS, Nishi S, et al. Aire-dependent thymic development of tumor-associated regulatory $\mathrm{T}$ cells. Science 2013;339:1219-24.

33. Jordan MS, Boesteanu A, Reed AJ, et al. Thymic selection of $\mathrm{CD} 4+\mathrm{CD} 25+$ regulatory $\mathrm{T}$ cells induced by an agonist self-peptide. Nat Immunol 2001;2:301-6.

34. Takeuchi $Y$, Nishikawa H. Roles of regulatory T cells in cancer immunity. Int Immunol 2016;28:401-9.

35. Miyara M, Sakaguchi S. Natural regulatory T cells: mechanisms of suppression. Trends Mol Med 2007;13:10816.

36. Wing K, Onishi Y, Prieto-Martin P, et al. CTLA-4 control over Foxp3+ regulatory T cell function. Science 2008;322:271-5.

37. Onishi $Y$, Fehervari Z, Yamaguchi T, et al. Foxp3+ natural regulatory $\mathrm{T}$ cells preferentially form aggregates on dendritic cells in vitro and actively inhibit their maturation. Proc Natl Acad Sci U S A 2008;105:10113-8.

38. Pandiyan $\mathrm{P}$, Zheng $\mathrm{L}$, Ishihara $\mathrm{S}$, et al. CD4+CD25+Foxp3+ regulatory $\mathrm{T}$ cells induce cytokine deprivation-mediated apoptosis of effector CD4+ T cells. Nat Immunol 2007;8:1353-62.

39. Sakaguchi S, Wing K, Onishi Y, et al. Regulatory T cells: how do they suppress immune responses? Int Immunol 2009;21:1105-11.

40. von Boehmer H, Daniel C. Therapeutic opportunities for manipulating $\mathrm{T}(\mathrm{Reg})$ cells in autoimmunity and cancer. Nat Rev Drug Discov 2013;12:51-63.

41. Chaudhary B, Elkord E. Regulatory T Cells in the Tumor Microenvironment and Cancer Progression: Role and Therapeutic Targeting. Vaccines (Basel) 2016;4:28 .

42. Ward ST, Li KK, Hepburn E, et al. The effects of CCR5 inhibition on regulatory T-cell recruitment to colorectal cancer. Br J Cancer 2015;112:319-28.

43. Tzankov A, Meier C, Hirschmann P, et al. Correlation of high numbers of intratumoral FOXP3+ regulatory $\mathrm{T}$ cells with improved survival in germinal center-like diffuse large B-cell lymphoma, follicular lymphoma and classical Hodgkin's lymphoma. Haematologica 2008;93:193-200.

44. Liu C, Workman CJ, Vignali DA. Targeting regulatory T cells in tumors. FEBS J 2016;283:2731-48.

45. Galvin KC, Dyck L, Marshall NA, et al. Blocking retinoic acid receptor- $\alpha$ enhances the efficacy of a dendritic cell vaccine against tumours by suppressing the induction of regulatory T cells. Cancer Immunol Immunother 2013;62:1273-82.

46. Facciabene A, Peng X, Hagemann IS, et al. Tumour hypoxia promotes tolerance and angiogenesis via CCL28 and T(reg) cells. Nature 2011;475:226-30.

47. Simpson TR, Li F, Montalvo-Ortiz W, et al. Fc-dependent depletion of tumor-infiltrating regulatory $\mathrm{T}$ cells codefines the efficacy of anti-CTLA-4 therapy against melanoma. J Exp Med 2013;210:1695-710.

48. Selby MJ, Engelhardt JJ, Quigley M, et al. Anti-CTLA-4 antibodies of IgG2a isotype enhance antitumor activity through reduction of intratumoral regulatory $\mathrm{T}$ cells. Cancer Immunol Res 2013;1:32-42.

49. Jarnicki AG, Lysaght J, Todryk S, et al. Suppression of antitumor immunity by IL-10 and TGF-beta-producing $T$ cells infiltrating the growing tumor: influence of tumor environment on the induction of CD4+ and CD8+ regulatory T cells. J Immunol 2006;177:896-904.

50. Akeus P, Langenes V, Kristensen J, et al. Tregcell depletion promotes chemokine production and accumulation of CXCR3(+) conventional T cells in intestinal tumors. Eur J Immunol 2015;45:1654-66.

51. Sato E, Olson SH, Ahn J, et al. Intraepithelial CD8+ tumor-infiltrating lymphocytes and a high CD8+/ regulatory $\mathrm{T}$ cell ratio are associated with favorable prognosis in ovarian cancer. Proc Natl Acad Sci U S A 2005;102:18538-43.

52. Gao Q, Qiu SJ, Fan J, et al. Intratumoral balance of regulatory and cytotoxic $\mathrm{T}$ cells is associated with prognosis of hepatocellular carcinoma after resection. J Clin Oncol 2007;25:2586-93.

53. Angelova $\mathrm{M}$, Charoentong $\mathrm{P}$, Hackl H, et al. Characterization of the immunophenotypes and antigenomes of colorectal cancers reveals distinct tumor escape mechanisms and novel targets for immunotherapy. Genome Biol 2015;16:64.

54. Lee Y, Awasthi A, Yosef N, et al. Induction and molecular signature of pathogenic TH17 cells. Nat Immunol 2012;13:991-9.

55. Gu C, Wu L, Li X. IL-17 family: cytokines, receptors and 
signaling. Cytokine 2013;64:477-85.

56. Tartour E, Fossiez F, Joyeux I, et al. Interleukin 17, a T-cell-derived cytokine, promotes tumorigenicity of human cervical tumors in nude mice. Cancer Res 1999;59:3698-704.

57. Numasaki M, Watanabe M, Suzuki T, et al. IL-17 enhances the net angiogenic activity and in vivo growth of human non-small cell lung cancer in SCID mice through promoting CXCR-2-dependent angiogenesis. J Immunol 2005;175:6177-89.

58. Nam JS, Terabe M, Kang MJ, et al. Transforming growth factor beta subverts the immune system into directly promoting tumor growth through interleukin-17. Cancer Res 2008;68:3915-23.

59. Zhu X, Mulcahy LA, Mohammed RA, et al. IL-17 expression by breast-cancer-associated macrophages: IL17 promotes invasiveness of breast cancer cell lines. Breast Cancer Res 2008;10:R95.

60. Wang L, Yi T, Kortylewski M, et al. IL-17 can promote tumor growth through an IL-6-Stat3 signaling pathway. J Exp Med 2009;206:1457-64.

61. Murugaiyan G, Saha B. Protumor vs antitumor functions of IL-17. J Immunol 2009;183:4169-75.

62. Su X, Ye J, Hsueh EC, et al. Tumor microenvironments direct the recruitment and expansion of human Th17 cells. J Immunol 2010;184:1630-41.

63. Martin-Orozco N, Muranski P, Chung Y, et al. T helper 17 cells promote cytotoxic $T$ cell activation in tumor immunity. Immunity 2009;31:787-98.

64. Ankathatti Munegowda M, Deng Y, Mulligan SJ, et al. Th17 and Th17-stimulated CD8(+) T cells play a distinct role in Th17-induced preventive and therapeutic antitumor immunity. Cancer Immunol Immunother 2011;60:1473-84.

65. Kryczek I, Banerjee M, Cheng P, et al. Phenotype, distribution, generation, and functional and clinical relevance of Th17 cells in the human tumor environments. Blood 2009; 114:1141-9.

66. Zhou Y, Toh ML, Zrioual S, et al. IL-17A versus IL-17F induced intracellular signal transduction pathways and modulation by IL-17RA and IL-17RC RNA interference in AGS gastric adenocarcinoma cells. Cytokine 2007;38:157-64.

67. Cantini G, Pisati F, Mastropietro A, et al. A critical role for regulatory $\mathrm{T}$ cells in driving cytokine profiles of Th17 cells and their modulation of glioma microenvironment. Cancer Immunol Immunother 2011;60:1739-50.
68. Qian X, Chen H, Wu X, et al. Interleukin-17 acts as double-edged sword in anti-tumor immunity and tumorigenesis. Cytokine 2017;89:34-44.

69. Pan B, Shen J, Cao J, et al. Interleukin-17 promotes angiogenesis by stimulating VEGF production of cancer cells via the STAT3/GIV signaling pathway in non-smallcell lung cancer. Sci Rep 2015;5:16053.

70. Xu B, Guenther JF, Pociask DA, et al. Promotion of lung tumor growth by interleukin-17. Am J Physiol Lung Cell Mol Physiol 2014;307:L497-508.

71. Knochelmann HM, Dwyer CJ, Bailey SR, et al. When worlds collide: Th17 and Treg cells in cancer and autoimmunity. Cell Mol Immunol 2018;15:458-69.

72. Boutros C, Tarhini A, Routier E, et al. Safety profiles of anti-CTLA-4 and anti-PD-1 antibodies alone and in combination. Nat Rev Clin Oncol 2016;13:473-86.

73. Menzies AM, Johnson DB, Ramanujam S, et al. AntiPD-1 therapy in patients with advanced melanoma and preexisting autoimmune disorders or major toxicity with ipilimumab. Ann Oncol 2017;28:368-76.

74. Francisco LM, Salinas VH, Brown KE, et al. PD-L1 regulates the development, maintenance, and function of induced regulatory T cells. J Exp Med 2009;206:3015-29.

75. Lohr J, Knoechel B, Abbas AK. Regulatory T cells in the periphery. Immunol Rev 2006;212:149-62.

76. Park HJ, Park JS, Jeong YH, et al. PD-1 upregulated on regulatory $\mathrm{T}$ cells during chronic virus infection enhances the suppression of CD8+ T cell immune response via the interaction with PD-L1 expressed on CD8+ T cells. J Immunol 2015;194:5801-11.

77. Brown JA, Dorfman DM, Ma FR, et al. Blockade of programmed death-1 ligands on dendritic cells enhances T cell activation and cytokine production. J Immunol 2003;170:1257-66.

78. Raimondi G, Shufesky WJ, Tokita D, et al. Regulated compartmentalization of programmed cell death-1 discriminates CD4+CD25+ resting regulatory $\mathrm{T}$ cells from activated T cells. J Immunol 2006;176:2808-16.

79. Wong M, La Cava A, Hahn BH. Blockade of programmed death-1 in young (New Zealand Black x New Zealand White)F1 mice promotes the suppressive capacity of CD4+ regulatory $\mathrm{T}$ cells protecting from lupus-like disease. $\mathrm{J}$ Immunol 2013;190:5402-10.

80. Lee YK, Turner H, Maynard CL, et al. Late developmental plasticity in the T helper 17 lineage. Immunity 2009;30:92107.

81. Basdeo SA, Cluxton D, Sulaimani J, et al. Ex-Th17 
(Nonclassical Th1) Cells Are Functionally Distinct from Classical Th1 and Th17 Cells and Are Not Constrained by Regulatory T Cells. J Immunol 2017;198:2249-59.

Cite this article as: Zhang $\mathrm{L}$, Zhang $\mathrm{M}, \mathrm{Xu} J$, Li S, Chen Y, Wang W, Yang J, Li S, Gu M. The role of the programmed cell death protein-1/programmed death-ligand 1 pathway, regulatory $\mathrm{T}$ cells and $\mathrm{T}$ helper 17 cells in tumor immunity: a narrative review. Ann Transl Med 2020;8(22):1526. doi: 10.21037/atm-20-6719
82. Esfahani K, Miller WH, Jr. Reversal of Autoimmune Toxicity and Loss of Tumor Response by Interleukin-17 Blockade. N Engl J Med 2017;376:1989-91. 